Pacific Journal of Mathematics

THE JACOBIAN OF A GROWTH TRANSFORMATION 


\title{
THE JACOBIAN OF A GROWTH TRANSFORMATION
}

\author{
D. S. PASSMAN
}

The transformation $T$, described in a paper of Baum and Eagon, is frequently a growth transformation which affords an iterative technique for maximizing certain functions. In this paper, the Jacobian matrix $J$ of $T$ is studied. It is shown, for example, that the eigenvalues of $J$ are real and nonnegative in a large number of cases. In addition, these eigenvalues are considered at critical points of $T$. One necessary assumption used throughout is that the function $P$ to be maximized is homogeneous in the variables involved.

The author would like to thank P. Stebe for a number of stimulating conversations and useful suggestions.

1. Notation. Let $P$ be a function of the variables $x_{i j}$ with domain of definition $D$ given by

$$
x_{i j}>0 \quad \text { and } \quad \sum_{j} x_{i j}=1 .
$$

Assume that on this domain both $P$ and all its partial derivatives $\partial P / \partial x_{i j}$ are positive. Moreover we assume that the second partial derivatives of $P$ exist and are continuous. Then the particular transformation $T$ of $P$ which we study here is given by (see [1])

$$
T\left(x_{i j}\right)=\frac{x_{i j} \partial P / \partial x_{i j}}{\sum_{k} x_{i k} \partial P / \partial x_{i k}} .
$$

Clearly $T$ maps $D$ into $D$.

We say that $P$ is row homogeneous if for each $i P$ is homogeneous of degree $w_{i}>0$ in the variables $x_{i 1}, x_{i 2}, \cdots$. In this case (1.1) simplifies by means of Euler's formula and we obtain

$$
T\left(x_{i j}\right)=\frac{x_{i j} \partial P / \partial x_{i j}}{w_{i} P} .
$$

Let us assume now that $P$ is row homogeneous. While the double subscript on the symbol $x_{i j}$ makes the domain $D$ easier to visualize, it turns out that a single subscript makes our later computations neater. Therefore we make the following notational change. Observe that the variables $\left\{x_{i j}\right\}$ are not all independent because of the constraints $\sum_{j} x_{i j}=1$. Thus in each row of the array $\left(x_{i j}\right)$ there is one variable which is dependent upon the others. Let us suppose now 
that there are a total of $n^{\prime}$ variables of which $n$ are independent. We can then write the variables $\left\{x_{i j}\right\}$ as

$$
x_{1}, x_{2}, \cdots, x_{n}, x_{n+1}, \cdots, x_{n}
$$

where $x_{1}, x_{2}, \cdots, x_{n}$ are independent and the remaining ones are dependent. Now the set $\left\{x_{n+1}, x_{n+2}, \cdots, x_{n^{\prime}}\right\}$ clearly contains precisely one variable from each row so we can certainly use the subscripts $n+1, n+2, \cdots, n^{\prime}$ to designate these rows. Finally we introduce the function

$$
f:\{1,2, \cdots, n\} \longrightarrow\left\{n+1, n+2, \cdots, n^{\prime}\right\}
$$

so that $f(i)$ indicates the row containing $x_{i}$.

In this single subscripted notation we see that for $i, j \leqq n, x_{i}$ and $x_{j}$ are in the same row if and only if $f(i)=f(j)$. Thus the fact that the sum of the variables in the row containing $x_{i}$ is 1 becomes

$$
1-x_{f(i)}=\sum_{j=1}^{n} \delta_{f(i) f(j)} x_{j} \quad \text { for } i \leqq n .
$$

Also setting $y_{i}=T\left(x_{i}\right)$ equation (1.2) now reads

$$
y_{i}=\frac{x_{i} \partial P / \partial x_{i}}{w_{f(i)} p} \quad \text { for } i \leqq n .
$$

Now suppose that $Q$ is a function of $x_{1}, \cdots, x_{n^{\prime}}$. Then we use as above $\partial Q / \partial x_{i}$ to denote the partial derivative of $Q$ with respect to $x_{i}$. On the other hand, $Q$ can be viewed as a function of the independent variables $x_{1}, \cdots, x_{n}$. If we do this, then we use $d Q / d x_{i}$ to denote the partial derivative of $Q$ with respect to $x_{i}$ for $i \leqq n$. It follows from (1.3) and the chain rule that

$$
\frac{d Q}{d x_{i}}=\frac{\partial Q}{\partial x_{i}}-\frac{\partial Q}{\partial x_{f(i)}} \quad \text { for } i \leqq n .
$$

The Jacobian of the growth transformation $T$ is the $n \times n$ matrix

$$
J=\left[\frac{d y_{i}}{d x_{j}}\right] \quad i, j \leqq n .
$$

It is the matrix which we plan to study.

2. Real eigenvalues. Let $N$ denote the $n \times n$ symmetric matrix 


$$
N=\left[\frac{x_{i}}{w_{f(i)}}\left(\delta_{i j}-\delta_{f(i) f(j)} x_{j}\right)\right] .
$$

The interplay of this matrix with $J$ will prove to be of fundamental importance.

Lemma $1 . \quad N$ is a positive definite matrix.

Proof. Since the ordering of the variables $x_{1}, x_{2}, \cdots, x_{n}$ does not effect the nature of $N$, we may assume that the variables are grouped together according to the row of the array $\left(x_{i j}\right)$ they are contained in. Then $N$ clearly becomes a block diagonal matrix with each block corresponding to a row of $\left(x_{i j}\right)$. Since it clearly suffices to show that each of these blocks is a positive definite matrix, it therefore suffices to consider the case in which $\left(x_{i j}\right)$ has only one row. Thus $n^{\prime}=n+1$ and

$$
w_{n+1} N=\left[\delta_{i j} x_{i}-x_{i} x_{j}\right] .
$$

Let $z$ be the real row vector $z=\left[z_{1}, z_{2}, \cdots, z_{n}\right] \neq 0$ and set

$$
\begin{aligned}
u & =\left[\sqrt{x_{1}}, \sqrt{x_{2}}, \cdots, \sqrt{x_{n}}\right] \\
v & =\left[\sqrt{x_{1}} z_{1}, \sqrt{x_{2}} z_{2}, \cdots, \sqrt{x_{n}} z_{n}\right] .
\end{aligned}
$$

Then using (, ) for the usual inner product of vectors we have

$$
\begin{aligned}
z\left(w_{n+1} N\right) z^{T} & =\sum_{1}^{n} x_{i} z_{i}^{2}-\left(\sum_{1}^{n} x_{i} z_{i}\right)^{2} \\
& =(v, v)-(u, v)^{2}>(u, u)(v, v)-(u, v)^{2} \geqq 0
\end{aligned}
$$

by Cauchy's inequality and the fact that

$$
(u, u)=x_{1}+x_{2}+\cdots+x_{n}=1-x_{n+1}<1 .
$$

The lemma is proved.

TheOREm 2. Let $P$ be a row homogeneous function. Then

$$
J N=\left[x_{j} / w_{f(j)} \partial y_{i} / \partial x_{j}\right]
$$

is a symmetric matrix.

Proof. From (1.4) it is clear that $y_{i}$ is row homogeneous of degree zero. Thus Euler's equation yields

$$
x_{f(j)} \partial y_{i} / \partial x_{f(j)}+\sum_{k=1}^{n} \delta_{f(j) f(k)} x_{k} \partial y_{i} / \partial x_{k}=0 \text {. }
$$

Let $J N=\left[h_{i j}\right]$. Then by (1.5) and the symmetry of $N$ we have 


$$
\begin{aligned}
w_{f(j)} h_{i j}= & \sum_{k=1}^{n} \frac{d y_{i}}{d x_{k}} \cdot x_{k}\left(\delta_{k j}-\delta_{f(k) f(j)} x_{j}\right) \\
= & x_{j} \frac{d y_{i}}{d x_{j}}-x_{j} \sum_{k=0}^{n} \delta_{f(k) f(j)} x_{k} \frac{d y_{i}}{d x_{k}} \\
= & x_{j}\left(\frac{\partial y_{i}}{\partial x_{j}}-\frac{\partial y_{i}}{\partial x_{f(j)}}\right)-x_{j} \sum_{k=1}^{n} \delta_{f(k) f(j)} x_{k} \frac{\partial y_{i}}{\partial x_{k}} \\
& +x_{j} \frac{\partial y_{i}}{\partial x_{f(j)}} \sum_{k=1}^{n} \delta_{f(k) f(j)} x_{k} \cdot
\end{aligned}
$$

Thus (2.2) and (1.3) ${ }_{35}^{\text {Ty }}$ yield

$$
\begin{aligned}
w_{f(j)} h_{i j}= & x_{j}\left(\frac{\partial y_{i}}{\partial x_{j}}-\frac{\partial y_{i}}{\partial x_{f(j)}}\right)+x_{j} x_{f(j)} \frac{\partial y_{i}}{\partial x_{f(j)}} \\
& +x_{j}\left(1-x_{f(j)}\right) \frac{\partial y_{i}}{\partial x_{f(j)}}=x_{j} \frac{\partial y_{i}}{\partial x_{j}} .
\end{aligned}
$$

Therefore we have

$$
h_{i j}=x_{j} / w_{f(j)} \partial y_{i} / \partial x_{j} \text {. }
$$

It remains to show that $h_{i j}=h_{j i}$ and to do this we may assume that $i \neq j$. Then by (1.4)

$$
h_{i j}=\frac{x_{i} x_{j}}{w_{f(i)} w_{f(j)} P^{2}}\left(P \partial^{2} P / \partial x_{i} \partial x_{j}-\left(\partial P / \partial x_{i}\right)\left(\partial P / \partial x_{j}\right)\right)
$$

so the result clearly follows.

Corollary 3. Let $P$ be a row homogeneous function. Then $J$ is diagonalizable and all eigenvalues of $J$ are real.

Proof. Write $J N=A$. Since $N$ is positive definite we have $N=Q Q^{T}$ for some real nonsingular matrix $Q$. Set $R=Q^{-1}$. Then we have easily

$$
R J R^{-1}=R A R^{T} .
$$

Since $R A R^{T}$ is real and symmetric by Theorem 2, it is diagonalizable with all real eigenvalues. Thus (2.3) yields the result.

3. Critical points. In this section we study in more detail the nature of $J$ at a critical point. It follows from (1.4) and (1.5) that at such a point we have $x_{i}=y_{i}$ and $\partial P / \partial x_{i}=w_{f(i)} P$. Recall that a critical point is a point at which

$$
\frac{d P}{d x_{i}}=0 \quad \text { for } i \leqq n .
$$


THEOREM 4. At a critical point we have

$$
J=I+\frac{N}{P}\left[\frac{d^{2} P}{d x_{i} d x_{j}}\right]
$$

where $I$ is the $n \times n$ identity matrix.

Proof. We start with Euler's equation for the row homogeneity of $P$. For $i \leqq n$ we have

$$
w_{f(i)} P=x_{f(i)} \partial P / \partial x_{f(i)}+\sum_{k=1}^{n} \delta_{f(i) f(k)} x_{k} \partial P / \partial x_{k}
$$

and differentiating this identity with respect to $x_{j}$ yields

$$
\begin{aligned}
w_{f(i)} \frac{d P}{d x_{j}}= & x_{f(i)} \frac{d}{d x_{j}} \partial P / \partial x_{f(i)}+\sum_{k=1}^{n} \delta_{f(i) f(k)} x_{k} \frac{d}{d x_{j}} \partial P / \partial x_{k} \\
& +\delta_{f(i) f(j)}\left(\partial P / \partial x_{j}-\partial P / \partial x_{f(i)}\right) .
\end{aligned}
$$

Observe that the last term is just $\delta_{f(i) f(j)} d P / d x_{j}$ so the above becomes

$$
\begin{aligned}
\left(w_{f(i)}-\delta_{f(i) f(j)}\right) \frac{d P}{d x_{j}}= & x_{f(i)} \frac{d}{d x_{j}} \partial P / \partial x_{f(i)} \\
& +\sum_{k=1}^{n} \delta_{f(i) f(k)} x_{k} \frac{d}{d x_{j}} \partial P / \partial x_{k} .
\end{aligned}
$$

Now at a critical point $d P / d x_{j}=0$ so

$$
0=x_{f(i)} \frac{d}{d x_{j}} \partial P / \partial x_{f(i)}+\sum_{k=1}^{n} \delta_{f(i) f(k)} x_{k} \frac{d}{d x_{j}} \partial P / \partial x_{k} .
$$

By (1.5) for $i, j \leqq n$

$$
\frac{d^{2} P}{d x_{i} d x_{j}}=\frac{d}{d x_{j}} \frac{\partial P}{\partial x_{i}}-\frac{d}{d x_{j}} \frac{\partial P}{\partial x_{f(i)}}
$$

and substituting

$$
\frac{d}{d x_{j}} \frac{\partial P}{\partial x_{k}}=\frac{d^{2} P}{d x_{k} d x_{j}}+\frac{d}{d x_{j}} \frac{\partial P}{\partial x_{f(k)}}
$$

into (3.2) yields

$$
\begin{aligned}
0= & x_{f(i)} \frac{d}{d x_{j}} \frac{\partial P}{\partial x_{f(i)}}+\sum_{k=1}^{n} \delta_{f(i) f(k)} x_{k} \frac{d}{d x_{j}} \frac{\partial P}{\partial x_{f(k)}} \\
& +\sum_{k=1}^{n} \delta_{f\left(\xi^{\prime}\right)^{\prime}(k)} x_{k} \frac{d^{2} P}{d x_{k} d x_{j}} .
\end{aligned}
$$

Now clearly 


$$
\delta_{f(i) f(k)} x_{k} \frac{d}{d x_{j}} \frac{\partial P}{\partial x_{f(k)}}=\delta_{f(i) f(k)} x_{k} \frac{d}{d x_{j}} \frac{\partial P}{\partial x_{f(i)}}
$$

so (3.4) becomes

$$
\begin{aligned}
0= & \frac{d}{d x_{j}} \frac{\partial P}{\partial x_{f(i)}}\left(x_{f(i)}+\sum_{k=1}^{n} \delta_{f(i) f(k)} x_{k}\right) \\
& +\sum_{k=1}^{n} \delta_{f(i) f(k)} x_{k} \frac{d^{2} P}{d x_{k} d x_{j}} .
\end{aligned}
$$

Hence by (1.3) we have

$$
\frac{d}{d x_{j}} \frac{\partial P}{i \partial x_{f(i)}}=-\sum_{k=1}^{n} \delta_{f(i) f(k)} x_{k} \frac{d^{2} P}{d x_{k} d x_{j}}
$$

We now compute $J$ at the critical point. By (1.4) and (3.1)

$$
\begin{aligned}
\frac{d y_{i}}{d x_{j}} & =\delta_{i j} \frac{\partial P / \partial x_{i}}{w_{f(i)} P}+\frac{x_{i}}{w_{f(i)} P} \frac{d}{d x_{j}} \frac{\partial P}{\partial x_{i}} \\
& =\delta_{i j}+\frac{x_{i}}{w_{f(i)} P} \frac{d}{d x_{j}} \frac{\partial P}{\partial x_{i}}
\end{aligned}
$$

since at a critical point $\partial P / \partial x_{i}=w_{f(i)} P$. Thus

$$
J=I+\frac{1}{P}\left[\frac{x_{i}}{w_{f(i)}} \frac{d}{d x_{j}} \frac{\partial P}{\partial x_{i}}\right] .
$$

Let $E=\left[e_{i j}\right]$ denote the latter matrix. Then using (3.3) and (3.5) we have

$$
\begin{aligned}
e_{i j} & =\frac{x_{i}}{w_{f(i)}}\left(\frac{d^{2} P}{d x_{i} d x_{j}}+\frac{d}{d x_{j}} \frac{\partial P}{\partial x_{f(i)}}\right) \\
& =\frac{x_{i}}{w_{f(i)}} \sum_{k=1}^{n}\left(\delta_{i k}-\delta_{f(i) f(k)} x_{k}\right) \frac{d^{2} P}{d x_{k} d x_{j}}
\end{aligned}
$$

and this is the $(i, j)$ th entry in the matrix product

$$
\left[\frac{x_{i}}{w_{f(i)}}\left(\delta_{i j}-\delta_{f(i) f(j)} x_{j}\right)\right]\left[\frac{d^{2} P}{d x_{i} d x_{j}}\right] \text {. }
$$

In view of (3.6), the result follows.

Let $B$ denote the matrix

$$
B=\left[\frac{d^{2} P}{d x_{i} d x_{j}}\right]
$$

COROLlary 5. Suppose that at a critical point we have det $B \neq 0$ and let $\lambda$ be an eigenvalue of $J$. Then 
(i) at a minimum, $\lambda>1$

(ii) at a maximum, $\lambda<1$.

Proof. By Theorem 4, $\lambda=1+\mu / P$ where $\mu$ is an eigenvalue of $N B$ and by Corollary $3, \lambda$ is real. Thus it suffices to show that $\mu$ is positive at a minimum and negative at a maximum.

Let $v$ be a real column eigenvector for $\mu$. Then $N B v=\mu v$ yields easily

$$
v^{T} B v=\mu\left(u^{T} N u\right)
$$

where $v=N u$. Since $N$ is positive definite by Lemma 1 we have $u^{T} N u>0$.

Now at a minimum, since $\operatorname{det} B \neq 0$, we see that $B$ is a positive definite matrix. Thus $v^{T} B v>0$ and $\mu>0$ by (3.8). Similarly at a maximum, $B$ is negative definite so $\mu<0$. This completes the proof.

4. Polynomials. We assume here that $P$ is a row homogeneous function and use the notation of the preceding sections. In addition, we assume that $P$ is a polynomial with positive coefficients so that (in single subscripted variables)

$$
P=\sum_{a} m_{a}
$$

where

$$
m_{a}=e_{a} x_{1}^{a_{1}} x_{2}^{a_{2}} \cdots x_{n^{\prime} n^{\prime}}^{a^{\prime}}, \quad e_{a}>0 .
$$

Here, of course, $a$ designates the $n^{\prime}$-tuple. $a=\left(a_{1}, a_{2}, \cdots, a_{n^{\prime}}\right)$. Let $\mathscr{A}$ denote the set of all such $a$ 's which occur in $P$.

Fix some ordering of the $a$ 's and let $\alpha$ denote a subset $\{a, b\}$ of $\mathscr{A}$ with $b>a$. For each such $\alpha$ set

$$
m_{\alpha}=m_{a} m_{b}, \quad \alpha_{i}=b_{i}-a_{i} .
$$

Since $P$ is row homogeneous we have for $i \leqq n$

$$
a_{f(i)}+\sum_{j=1}^{n} \delta_{f(i) f(j)} a_{j}=w_{f(i)}
$$

and hence (4.3) yields

$$
\alpha_{f(i)}+\sum_{j=1}^{n} \delta_{f(i) f(j)} \alpha_{j}=0 .
$$

For the row homogeneous polynomial $P$ we define the vector subspace $V(P) \subseteq R^{n^{\prime}}$ to be the subspace of $R^{n^{\prime}}$ spanned by all $n^{\prime}$ tuples $\left(\alpha_{1}, \alpha_{2}, \cdots, \alpha_{n^{\prime}}\right)$ with $\alpha=\{a, b\}, a, b \in \mathscr{A}$. In view of (4.4) we 
have certainly

$$
\operatorname{dim} V(P) \leqq n
$$

THEOREM 6. Let $P$ be a row homogeneous polynomial. Then $J N$ is a positive semi-definite symmetric matrix with

$$
\operatorname{rank} J N=\operatorname{dim} V(P) .
$$

Proof. Let $P$ be given by (4.1) and (4.2). Then by (1.4)

$$
w_{f(i)} y_{i}=\frac{\Sigma a_{i} m_{a}}{\Sigma m_{a}}
$$

and we have

$$
\begin{aligned}
w_{f(i)} P^{2} x_{j} \partial y_{i} / \partial x_{j} & =\left(\sum_{a} m_{a}\right)\left(\sum_{b} b_{i} b_{j} m_{b}\right)-\left(\sum_{a} a_{j} m_{a}\right)\left(\sum_{b} b_{i} m_{b}\right) \\
& =\sum_{a, b} m_{a} m_{b}\left(b_{i} b_{j}-b_{i} a_{j}\right) .
\end{aligned}
$$

Observe that the inner summand vanishes at $a=b$. Thus if we sum over $a<b$ then we obtain

$$
\begin{aligned}
w_{f(i)} P^{2} x_{j} \frac{\partial y_{i}}{\partial x_{j}} & =\sum_{a<b} m_{a} m_{b}\left(b_{i} b_{j}-b_{i} \alpha_{j}+a_{i} a_{j}-a_{i} b_{j}\right) \\
& =\sum_{\alpha} m_{\alpha} \alpha_{i} \alpha_{j}
\end{aligned}
$$

in the notation of (4.3). Thus by Theorem 2

$$
P^{2} J N=\left[\sum_{\alpha} m_{\alpha} \alpha_{i} \alpha_{j} / w_{f(i)} w_{f(j)}\right]
$$

Let $z=\left[z_{1}, z_{2}, \cdots, z_{n}\right]$ be a row vector of real entries. Then

$$
\begin{aligned}
z\left(P^{2} J N\right) z^{T} & =\sum_{i, j} \sum_{\alpha} m_{\alpha} \alpha_{i} \alpha_{j} z_{i} z_{j} / w_{f(i)} w_{f(j)} \\
& =\sum_{\alpha} m_{\alpha}\left(\sum_{i} \alpha_{i} z_{i} / w_{f(i)}\right)^{2} .
\end{aligned}
$$

Thus clearly $P^{2} J N$ and hence $J N$ is positive semi-definite.

It remains to compute the rank of $J N$. Let $W(P) \subseteq R^{n}$ be the subspace of $R^{n}$ spanned by all $n$-tuples $\tilde{\alpha}=\left(\alpha_{1}, \alpha_{2}, \cdots, \alpha_{n}\right)$. In view of (4.4) we have clearly

$$
\operatorname{dim} W(P)=\operatorname{dim} V(P) \text {. }
$$

Let [,] be the inner product on $R^{n}$ defined by

$$
\left[\left(u_{1}, u_{2}, \cdots, u_{n}\right),\left(v_{1}, v_{2}, \cdots, v_{n}\right)\right]=\sum_{i=1}^{n} u_{i} v_{i} / w_{f(i)} .
$$


Then (4.6) becomes

$$
z\left(P^{2} J N\right) z^{T}=\sum_{\alpha} m_{\alpha}[\tilde{\alpha}, z]^{2}
$$

Thus $z\left(P^{2} J N\right) z^{T}=0$ if and only if $z \in W(P)^{\perp}$, the orthogonal compliment of $W(P)$. Finally since $P^{2} J N$ is positive semi-definite we have

$$
\operatorname{rank} P^{2} J N=n-\operatorname{dim} W(P)^{\prime}=\operatorname{dim} W(P)
$$

and the result follows from (4.7).

CoRollary 7. Let $P$ be a row homogeneous polynomial. Then all eigenvalues of $J$ are non-negative real numbers and hence $\operatorname{det} J \geqq 0$.

Proof. This follows immediately from (2.3) and Theorem 6.

Observe that Theorem 6 implies that $\operatorname{det} J>0$ if $\operatorname{dim} V(P)=n$ and $\operatorname{det} J=0$ otherwise. This fact is an unpublished result of L. Baum.

5. Examples. In this section, we consider a number of examples with $P$ not homogeneous. Suppose $y_{1}$ is given by

$$
y_{1}=\frac{x_{1} \partial P / \partial x_{1}}{x_{1} \partial P / \partial x_{1}+x_{2} \partial P / \partial x_{2}}
$$

Then

$$
\frac{1}{1-y_{1}}=1+\frac{x_{1} \partial P / \partial x_{1}}{x_{2} \partial P / \partial x_{2}}
$$

Differentiating with respect to some variable $x$ then yields

$$
\frac{d y_{1}}{d x}=\left(1-y_{1}\right)^{2} \frac{d}{d x}\left(\frac{x_{1} \partial P / \partial x_{1}}{x_{2} \partial P / \partial x_{2}}\right) \text {. }
$$

This formula enables the following computations to be done easily.

Let $P\left(x_{1}, x_{2}\right)=x_{1}+x_{1}^{2} x_{2}$ 。 Then

$$
\operatorname{det} J=\frac{d y_{1}}{d x_{1}}=\frac{\left(1-y_{1}\right)^{2}}{\left(x_{1} x_{2}\right)^{2}}\left(2 x_{1}-1\right)
$$

and this changes sign at $x_{1}=1 / 2$. Thus Corollary 7 requires that $P$ be homogeneous.

Now let

$$
P\left(x_{1}, x_{2}, x_{3}, x_{4}\right)=x_{1} x_{2}^{2}+x_{1}^{2} x_{3}+x_{2}^{2} x_{4}
$$


subject to the constraints $x_{1}+x_{3}=1, x_{2}+x_{4}=1$. Then

$$
J=\left[\begin{array}{ll}
\frac{\left(1-y_{1}\right)^{2}}{x_{1}^{2} x_{3}^{2}} x_{2}^{2}\left(2 x_{1}-1\right) & \frac{\left(1-y_{1}\right)^{2} 2 x_{2}}{x_{1} x_{3}} \\
\frac{\left(1-y_{2}\right)^{2} 2}{x_{4}} & \frac{\left(1-y_{2}\right)^{2} 2 x_{1}}{x_{4}^{2}}
\end{array}\right] .
$$

Thus

$$
\frac{x_{4}^{2}}{\left(1-y_{1}\right)^{2}\left(1-y_{2}\right)^{2}} \operatorname{det} J=\left|\begin{array}{ll}
\frac{x_{2}^{2}}{x_{1}^{2} x_{3}^{2}}\left(2 x_{1}-1\right) & \frac{2 x_{2}}{x_{1} x_{3}} \\
2 x_{4} & 2 x_{1}
\end{array}\right| .
$$

Finally let $x_{2} \sim 1$ so $x_{4} \sim 0$ and the right hand determinant is approximately equal to

$$
\frac{2\left(2 x_{1}-1\right)}{x_{1} x_{3}^{2}}
$$

which changes sign at $x_{1}=1 / 2$. Thus we see that even though $P$ is homogeneous, Corollary 7 can still fail unless $P$ is row homogeneous.

\section{REFERENCES}

1. L. Baum and J. Eagon, An inequality with application to statistical estimation for probabilistic functions of Markov processes and to a model for ecology, Bull. Amer. Math. Soc., 73 (1967), 360-363.

Received August 31, 1971.

Institute for Defense Analyses

PRINCETON, NEW JERSEY

AND

UNIVERSITY OF WISCONSIN, MADISON 


\section{PACIFIC JOURNAL OF MATHEMATICS}

\section{EDITORS}

H. SAMELSON

Stanford University

Stanford, California 94305

C. R. Hовву

University of Washington

Seattle, Washington 98105
J. DugundJI

Department of Mathematics University of Southern California

Los Angeles, California 90007

RICHARD ARENS

University of California

Los Angeles, California 90024

\section{ASSOCIATE EDITORS}

E. F. BECKENBACH

B. H. NeumanN

F. WOLF

K. YoSHIDA

\section{SUPPORTING INSTITUTIONS}

UNIVERSITY OF BRITISH COLUMBIA

CALIFORNIA INSTITUTE OF TECHNOLOGY

UNIVERSITY OF CALIFORNIA

MONTANA STATE UNIVERSITY

UNIVERSITY OF NEVADA

NEW MEXICO STATE UNIVERSITY

OREGON STATE UNIVERSITY

UNIVERSITY OF OREGON

OSAKA UNIVERSITY
UNIVERSITY OF SOUTHERN CALIFORNIA

STANFORD UNIVERSITY

UNIVERSITY OF TOKYO

UNIVERSITY OF UTAH

WASHINGTON STATE UNIVERSITY

UNIVERSITY OF WASHINGTON

AMERICAN MATHEMATICAL SOCIETY

NAVAL WEAPONS CENTER

Printed in Japan by International Academic Printing Co., Ltd., Tokyo, Japan 


\section{Pacific Journal of Mathematics}

\section{Vol. 44, No. $1 \quad$ May, 1973}

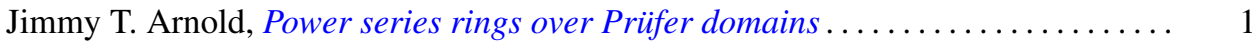

Maynard G. Arsove, On the behavior of Pincherle basis functions . . . . . . . . . 13

Jan William Auer, Fiber integration in smooth bundles ................. 33

George Bachman, Edward Beckenstein and Lawrence Narici, Function algebras

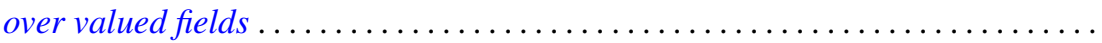

Gerald A. Beer, The index of convexity and the visibility function . . . . . . . . . . .

James Robert Boone, A note on mesocompact and sequentially mesocompact

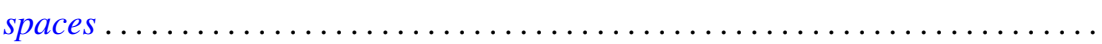

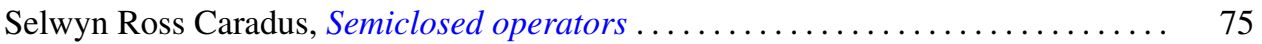

John H. E. Cohn, Two primary factor inequalities . . . . . . . . . . . . . . . 81

Mani Gagrat and Somashekhar Amrith Naimpally, Proximity approach to

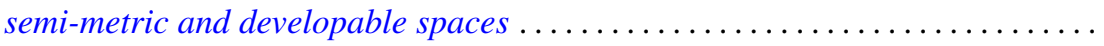

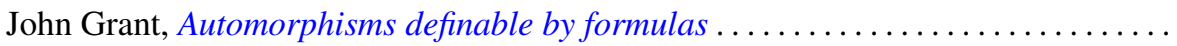

Walter Kurt Hayman, Differential inequalities and local valency ..............

Wolfgang H. Heil, Testing 3-manifolds for projective planes . . . . . . . . . . . . .

107

Melvin Hochster and Louis Jackson Ratliff, Jr., Five theorems on Macaulay

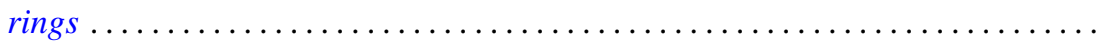

Thomas Benton Hoover, Operator algebras with reducing invariant subspaces ....

James Edgar Keesling, Topological groups whose underlying spaces are separable

Fréchet manifolds...

Frank Leroy Knowles, Idempotents in the boundary of a Lie group . .

191

George Edward Lang, The evaluation map and EHP sequences ...

201

Everette Lee May, Jr, Localizing the spectrum . . . . . . . . . . . .

211

Frank Belsley Miles, Existence of special $K$-sets in certain locally compact abelian groups.

Susan Montgomery, A generalization of a theorem of Jacobson. II . .

T. S. Motzkin and J. L. Walsh, Equilibrium of inverse-distance forces in

three-dimensions.

Arunava Mukherjea and Nicolas A. Tserpes, Invariant measures and the converse

of Haar's theorem on semitopological semigroups .

James Waring Noonan, On close-to-convex functions of order $\beta$

Donald Steven Passman, The Jacobian of a growth transformation

Dean Blackburn Priest, A mean Stieltjes type integral ........ .

Joe Bill Rhodes, Decomposition of semilattices with applications to topological

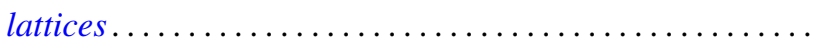

Claus M. Ringel, Socle conditions for $\mathrm{QF}-1$ rings ..........

Richard Rochberg, Linear maps of the disk algebra

Roy W. Ryden, Groups of arithmetic functions under Dirichlet convolution . .

Michael J. Sharpe, A class of operators on excessive functions

Erling Stormer, Automorphisms and equivalence in von Neumann algebras ..

Philip C. Tonne, Matrix representations for linear transformations on series

analytic in the unit disc. 